\title{
Performance evaluation of feature detection and feature matching for stereo visual odometry using SIFT and SURF
}

\begin{abstract}
Feature detection and feature matching are the most crucial parts in visual odometry process. In order to suit the real time process in visual odometry, both of the stages must be robust but at the same time are fast to compute. This paper presents the evaluation of Scale Invariant Feature Transform (SIFT) and Speeded Up Robust Feature (SURF) performances. The results show that SURF is outperform than SIFT in term of rate of matched points and also in computational time.
\end{abstract}

Keyword: Visual odometry; SIFT; SURF; Feature detection; Feature matching 